\title{
MiR-18a Inhibits PI3K/AKT Signaling Pathway to Regulate PDGF-BB-Induced Airway Smooth Muscle Cell Proliferation and Phenotypic Transformation
}

\author{
Wei YANG ${ }^{1,2}$, Yunbin CHEN $^{3}$, Chao HUANG ${ }^{4}$, Wenjian WANG ${ }^{5}$, Congfu HUANG ${ }^{6}$, \\ Yuanguang $\mathrm{LI}^{7}$
}

${ }^{1}$ Department of Pediatrics, The First Affiliated Hospital, Jinan University, Guangzhou, China, ${ }^{2}$ Department of Pediatrics, The Second Affiliated Hospital of Shenzhen University (The People's Hospital of Shenzhen Baoan District), Shenzhen, China, ${ }^{3}$ Department of Pediatrics, Guangdong Women's and Children's Hospital, Guangzhou, China, ${ }^{4}$ Department of Traditional Chinese Medicine, The Second Affiliated Hospital of Shenzhen University (The People's Hospital of Shenzhen Baoan District), Shenzhen, China. ${ }^{5}$ Department of Respiratory Medicine, Shenzhen Children's Hospital, Shenzhen, China, ${ }^{6}$ Department of Pediatrics, Longgang District Maternity \& Child Healthcare Hospital, Shenzhen, China, ${ }^{7}$ Department of Respiratory Medicine, The Second Affiliated Hospital of Shenzhen University (The People's Hospital of Shenzhen Baoan District), Shenzhen, China.

Received June 30, 2021

Accepted September 10, 2021

Epub Ahead of Print October 30, 2021

\section{Summary}

The increased proliferation and migration of airway smooth muscle cells (ASMCS) is a key process in the formation of airway remodeling in asthma. In this study, we focused on the expression of mircoRNA-18a (miR-18a) in airway remodeling in bronchial asthma and its related mechanisms. ASMCs are induced by platelet-derived growth factor BB (PDGF-BB) for in vitro airway remodeling. The expression of miR-18a in sputum of asthmatic patients and healthy volunteers was detected by qRT-PCR. The expression of miR-18a was over-expressed or interfered with in PDGF-BB-treated ASMCs. Cell proliferation, apoptosis and migration were detected by MTT, flow cytometry and Transwell, respectively, the expression of contractile phenotype marker proteins (SM-22a, a-SM-actin, calponin) and key molecules of the phosphatidylinositol 3-kinase (PI3K)/AKT pathway (PI3K, p-PI3K, AKT and p-AKT) in ASMCs were detected by Western blot. The expression of miR-18a was down-regulated in the sputum and PDGF-BB-treated ASMCs of asthma patients. PDGF-BB could promote the proliferation and migration of ASMCS and inhibit their apoptosis, it could also promote the phenotypic transformation of ASMCs and activate the PI3K/AKT pathway. MiR-18a could inhibit the proliferation, migration ability and phenotypic transformation of ASMCs induced by PDGF-BB to a certain extent and alleviate the effect of PDGF-BB in supressing apoptosis, while miR-18a could inhibit the activation of the PI3K/AKT pathway. MiR-18a inhibits PDGF-BB-induced proliferation, migration and phenotypic conversion of ASMCs by inhibiting the PI3K/AKT pathway, thus attenuating airway remodeling in asthma.

\section{Key words}

miR-18a • PDGF-BB • Airway smooth muscle cells (ASMCs) • Airway remodelingv $\bullet$ PI3K/AKT pathway

\section{Corresponding author}

Wei Yang and Yunbin Chen, Department of Pediatrics, The First Affiliated Hospital, Jinan University, Guangzhou, Guangdong 510630, China. Email: robertyung@163.com and chenyunbin9341@163.com

\section{Introduction}

Bronchial asthma is a complex respiratory syndrome caused by various pathological mechanisms. The main clinical manifestations include chronic airway inflammation, airway hyper-reactivity, reversible airflow

PHYSIOLOGICAL RESEARCH • ISSN 1802-9973 (online) - an open access article under the CC BY-NC-ND 4.0 license (C) 2021 Institute of Physiology of the Czech Academy of Sciences, Prague, Czech Republic Fax +420 241062 164, e-mail: physres@fgu.cas.cz, www.biomed.cas.cz/physiolres 
limitation, airway remodeling, and recurrent wheezing, cough, shortness of breath, and chest distress (KingBiggs 2019). Airway remodeling is an important pathological feature of asthma as a repair response to persistent inflammation (Hirota and Martin 2013). Most of current understanding of airway remodeling comes from studies of allergic asthma (Samitas et al. 2018). Airway remodeling in allergic bronchial asthma is thought to be the result of a chronic inflammatory response, mainly manifested by permanent airway tissue destruction and chronic tissue repair (Fehrenbach et al. 2017). However, there is growing evidence that asthma is a syndrome composed of multiple phenotypes/endotypes (Fehrenbach et al. 2017). Therefore, it is unknown whether the salient features of airway remodeling are associated with certain phenotypic/endotypic specificities.

Airway smooth muscle plays an important role in the lung. Hyperplastic and hypertrophic smooth muscle not only leads to airway wall thickening, causing airway stenosis but also reduces the contractility of the airway wall, increasing extracellular matrix synthesis and aggravating the fibrosis of the tracheal wall. Excessive proliferation and migration of airway smooth muscle can directly contribute to airway remodeling (Guida and Riccio 2019). Airway smooth muscle cells (ASMCs), fibroblasts and myofibroblasts are the main effector cells of airway subepithelial thickening in asthma, fibroblastto-myofibroblasttransition (FMT) is one of the main mechanisms of early airway remodeling (Michalik et al. 2018). In the pathogenesis of asthma, myofibroblasts do not initiate apoptosis immediately after completing their normal physiological functions, but rather persist in tissues, inducing contraction of surrounding cells and extracellular matrix (ECM), thus leading to secretion of growth factors and ECM components. Therefore, myofibroblasts have an impact on contractile and metabolic activities and participate in airway remodeling (Bretherton et al. 2020). Previous studies on FMT in asthma have identified a variety of precipitating factors, of which humoral factors including growth factors, cytokines and chemokines, play an important role in phenotypic transformation. Among them, platelet-derived growth factor BB (PDGF-BB) has been demonstrated to induce proliferation and migration of ASMCs and exacerbate airway remodeling (Dai et al. 2018). Due to the complex pathogenesis of asthma, factors may interact with each other and form corresponding feedback loops to further the transformation. Therefore, studying the interaction of various regulatory factors of FMT, thus identifying specific therapeutic targets, is an important direction in the treatment of irreversible airway remodeling.

The role of microRNA (miRNA) in regulating human biological functions and diseases has been a hot topic in research. MiRNAs are single-stranded non-coding RNAs containing only 19-22 bases of singlestranded small ribonucleic acid molecules. MiRNAs inhibit gene translation by binding to the mRNA3untranslated region (3'-UTR), which in turn negatively regulates gene expression (Inui et al. 2010, Vishnoi and Rani 2017). Studies have reported that miRNAs are involved in many biological processes, including stress and inflammatory regulation of host immune cells (Pua and Ansel 2015). An increasing number of studies have confirmed that miRNAs also play a regulatory role in asthma airway remodeling. Shao et al found that niRNA-133a can target and regulate insulin-like growth factor 1 receptors (IGF1R) and alleviate airway remodeling in asthma by inhibiting the phosphatidylinositol 3-kinase (PI3K)/AKT/mammalian target of rapamycin (mTOR) signaling pathway activation (Shao et al. 2019). Lou et al found that miR-192-5p attenuates airway remodeling and autophagy in asthma by targeting matrix metalloproteinase- 16 (MMP-16) and autophagy-related gene 7 (ATG7) (Lou et al. 2020). Some studies have found that miR-18a is down-regulated in nasal biopsies of asthmatic patients (Suojalehto et al. 2014). MiR-18a is also involved in the regulation of multiple signaling pathways (Yang et al. 2018, Ye et al. 2019, Yuan et al. 2019, Zhang et al. 2018) and regulates multiple downstream target genes to exert effects. However, the specific mechanism of action of miR-18a regulation in early airway remodeling in asthma is unknown. Therefore, in the present study, we measured the expression of miR-18a after PDGF-BB-induced ASMCs. We also explored the role of PI3K/AKT signaling pathway and miR-18a in early airway remodeling in cell model, laying a foundation for identifying a key therapeutic target for early intervention of irreversible airway remodeling.

\section{Methods and Materials}

\section{Subjects}

This study recruited 20 patients with bronchial asthma and 20 healthy controls. In the asthma group, there were 12 males and 8 females, aged $14-80$ years, all 
of whom visited the Outpatient and Inpatient Department of Respiratory and Critical Care of the Second Affiliated Hospital of Shenzhen University between 2018 and 2021. Inclusion criteria included medical history, physical examination, and physiological examination consistent with the national asthma control guidelines for asthma diagnosis. Exclusion criteria included history of smoking, chronic lung disease other than asthma within the past year, other serious chronic diseases, including congestive heart failure, chronic kidney disease, liver disease, and viral infections.

A total of 20 patients were enrolled in the control group (Normal group). All healthy controls (8 males and 12 females, aged 18-58 years) underwent a physical examination at our hospital during the same period as above, with good health and no history of asthma, allergic rhinitis or other allergic diseases. All subjects were non-smokers. This study was approved by the Ethics Committee of the People's Hospital of Shenzhen Baoan District, and all participants provided written informed consent (Approval Number: 2021042112015753).

\section{Sputum sample}

Subjects' first sputum in the morning was collected. The subjects were asked to gargle with water for $10 \mathrm{~min}$ before sputum collection and deep sputum was collected. Each sample must contain over $3 \mathrm{ml}$ of sputum to avoid saliva and nasal secretions. When transfer sputum specimen, the sample should be kept at a low temperature on ice. The collected sputum samples were transferred into a $50 \mathrm{~mL}$ centrifuge tube, added with cell washings $(30 \mathrm{~mL} / \mathrm{subject})$ and Dithiothreitol (DTT, $6 \mathrm{mM}, 1 \mathrm{~mL}$ ), and shaken on a shaker until the sputum was completely digested. The sample was then centrifuged at $2000 \mathrm{rpm}$ for $10 \mathrm{~min}$, the supernatant was discarded, and the cells at the bottom were transferred to the cell preservative for future use.

\section{Cell culture and processing}

ASMCs were purchased from the American type culture collection (ATCC, Virginia, USA). Cell cultures were maintained in high-glucose dulbecco's modified eagle medium (DMEM, ScienCell, CA, USA) containing $10 \%$ Fetal Bovine Serum (FBS, ScienCell, California, USA) at $37{ }^{\circ} \mathrm{C}$ in a humidified $5 \% \mathrm{CO}_{2}$ incubator.

MiR-18a mimics, inhibitor and negative control (NC, $100 \mathrm{nM}$ ) were purchased from Jima Gene Company (Shanghai, China). Transfection was performed using
Lipofectamine2000 reagent (Invitrogen, Carlsbad, CA, USA), and cells were transfected according to the instructions when they grew to 70-80\% confluence in six-well plates. The corresponding expression levels and functional assays were performed 24 hours after transfection.

Proliferation and migration of ASMCs were induced using PDGF-BB (PeproTech, Cranbury, NJ, USA). Cell grouping were named as follows: sham group: without any treatment, PDGF-BB group: $20 \mathrm{ng} / \mathrm{ml}$ PDGF-BB-induced cells, PDGF-BB + mi-NC group: PDGF-BB-induced cells and transfected mimic $\mathrm{NC}$, PDGF-BB + mi-miR-18a group: PDGF-BB-treated cells and transfected miR-18a mimic, PDGF-BB + in-NC group: PDGF-BB-treated cells and transfected inhibitor NC, PDGF-BB + in-miR-18a group: PDGF-BB-treated cells and transfected miR-18a inhibitor.

The PI3K/AKT pathway agonist IGF-1 (insulinlike growth factors-1, O2O, Israel) was used at a concentration of $10 \mu \mathrm{g} / \mathrm{mL}$. In the PDGF-BB + mi-miR$18 \mathrm{a}+$ IGF-1 group, cells were treated with PDGF-BB and IGF-1 and transfected with miR-18a mimic.

\section{$q R T-P C R$}

Total RNA was extracted using TRIZOL (Invitrogen, Carlsbad, CA, USA) from collected cells. Reverse transcription from RNA to cDNA was performed using a reverse transcription kit (TaKaRa, Tokyo, Japan), according to the instructions of the kit. Expression of genes was detected using a LightCycler480 (Roche, Indianapolis, IN, USA) quantitative fluorescence PCR instrument, and reaction conditions were set according to the operating instructions of the quantitative fluorescence PCR kit (SYBRGreenMix, RocheDiagnostics, Indianapolis, IN). U6 was adopted as the internal reference. Data analysis was performed using the $2^{-\Delta \Delta \mathrm{Ct}}$ method. The miR-18a and U6 primers used for qRT-PCR were purchased from GeneCopoeia Company (MD, USA).

\section{MTT assay}

The cell suspension was inoculated into a 96-well plate (100 $\mu \mathrm{L} /$ well, 2500 cells/well), and the plate was pre-cultured with $5 \% \mathrm{CO}_{2}$ at $37{ }^{\circ} \mathrm{C}$ for $24 \mathrm{~h}$, $48 \mathrm{~h}$ and $72 \mathrm{~h}$ respectively. $10 \mu \mathrm{L}$ MTT solution was added to each well. The culture plate was returned to the incubator for another $2 \mathrm{~h}$. After that, the opticaldensity (OD) at $570 \mathrm{~nm}$ was measured with a microplate reader. The test was repeated three times. 


\section{Annexin V-FITC/PI}

Cells with a confluence of $80-90 \%$ were digested and neutralized with EDTA-free trypsin, centrifuged at $2000 \mathrm{rpm}$ for $5 \mathrm{~min}$, and washed twice with precooled PBS, the supernatant was discarded. Prepared according to the instructions of the kit, $5 \mu \mathrm{L}$ of PI and FITC-labeled Annexin V was added to the cells which was then mixed well and reacted at room temperature in the shade for $15 \mathrm{~min}$, finally tested.

\section{Transwell}

Sterile transwell inserts were placed into 24-well plate wells, $100 \mu \mathrm{l}$ of equal cell concentration was added to the upper chamber of the inserts, and $800 \mu \mathrm{l}$ of complete medium containing $10 \%$ FBS was added to the lower chamber, then placed in the incubator for another $12 \mathrm{~h}$. After incubation, the transwell inserts were gently washed three times with PBS and subsequently adding in $4 \%$ paraformaldehyde and fixed for $30 \mathrm{~min}$. Non-migrated cells in the upper chamber were gently wiped away with a cotton swab and washed three times with PBS. After $0.4 \%$ crystal violet staining, five randomly selected fields were photographed under a microscope, the number of cells in each field was counted. Image $\mathrm{J}$ was used for statistical analysis.

\section{Western blot}

Cell lysis performed with RIPA lysis solution (Beyotime, Shanghai, China) to obtain cellular protein. The protein concentration was measured with the $\mathrm{BCA}$ kit (Beyotime). After membrane transfer, they were blocked in blocking solution for $60 \mathrm{~min}$ at room temperature and incubated with primary antibodies $\beta$-actin (4970S, Cell Signaling Technology, Boston, USA), SM-22 $\alpha$ (ab14106, Abcam, Mass., USA), $\alpha$-SM-actin (A5288, Sigma, St. Louis, Missouri, USA), calponin (17819S, Cell Signaling, Boston, USA), PI3K (4249S, Cell Signaling Technology), p-PI3K (17366s, Cell Signaling Technology), AKT (9272S, Cell Signaling Technology), p-AKT (4060S, Cell Signaling Technology) at $4{ }^{\circ} \mathrm{C}$ overnight. The next day, secondary antibody (horseradish peroxidase-labelled goat anti-rabbit $\mathrm{IgG}$, Beijing Kangwei Century Biotechnology Co., Ltd., China, Beijing) and incubated for 1 hour at room temperature and finished with three 10 min washes. After adding the developer to the membrane, the detection was performed using a chemiluminescence imaging system (Bio-rad).

\section{Statistical analysis}

Statistical analysis was performed by SPSS 24.0 software. Statistical data were expressed as mean \pm standard deviation (SD). One-way analysis of variance (ANOVA) or Student's t-test was used to compare more than two groups or two groups. Each experiment was triplicated. $\mathrm{P}<0.05$ indicated a significant difference.

\section{Results}

Down-regulation of miR-18a expression in the sputum of an asthma patient and PDGF-BB-induced ASMCS

MiR-18a expressions in the sputum of asthmatic patients examined by qRT-PCR $(\mathrm{P}<0.001)$ was noticeably lower than that of healthy volunteers (Fig. 1A). Further,
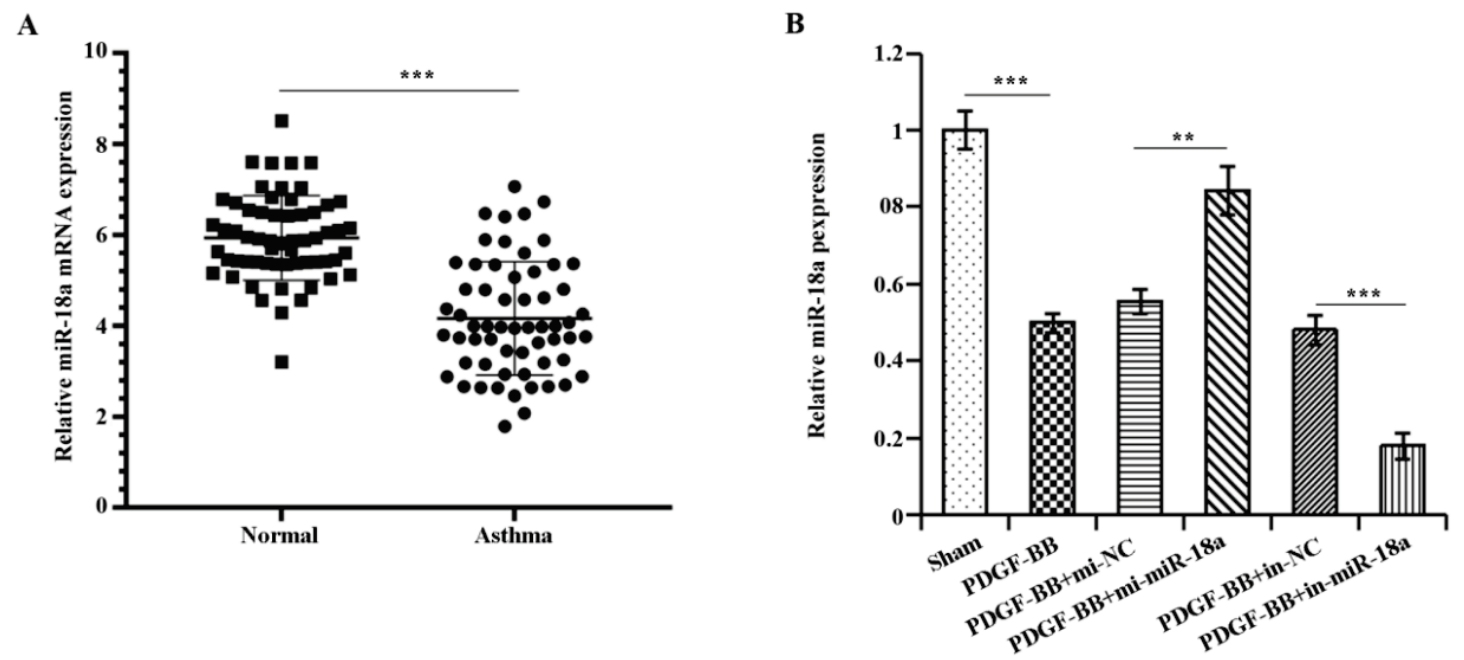

Fig. 1. MiR-18a expression was down-regulated in the sputum of an asthma patient and PDGF-BB-interfered ASMCs.

A) qRT-PCR results on miR-18a expression in normal and asthma subjects, B) qRT-PCR results on miR-18a expression in the experimental group. $* * \mathrm{P}<0.01, * * * \mathrm{P}<0.001$. 

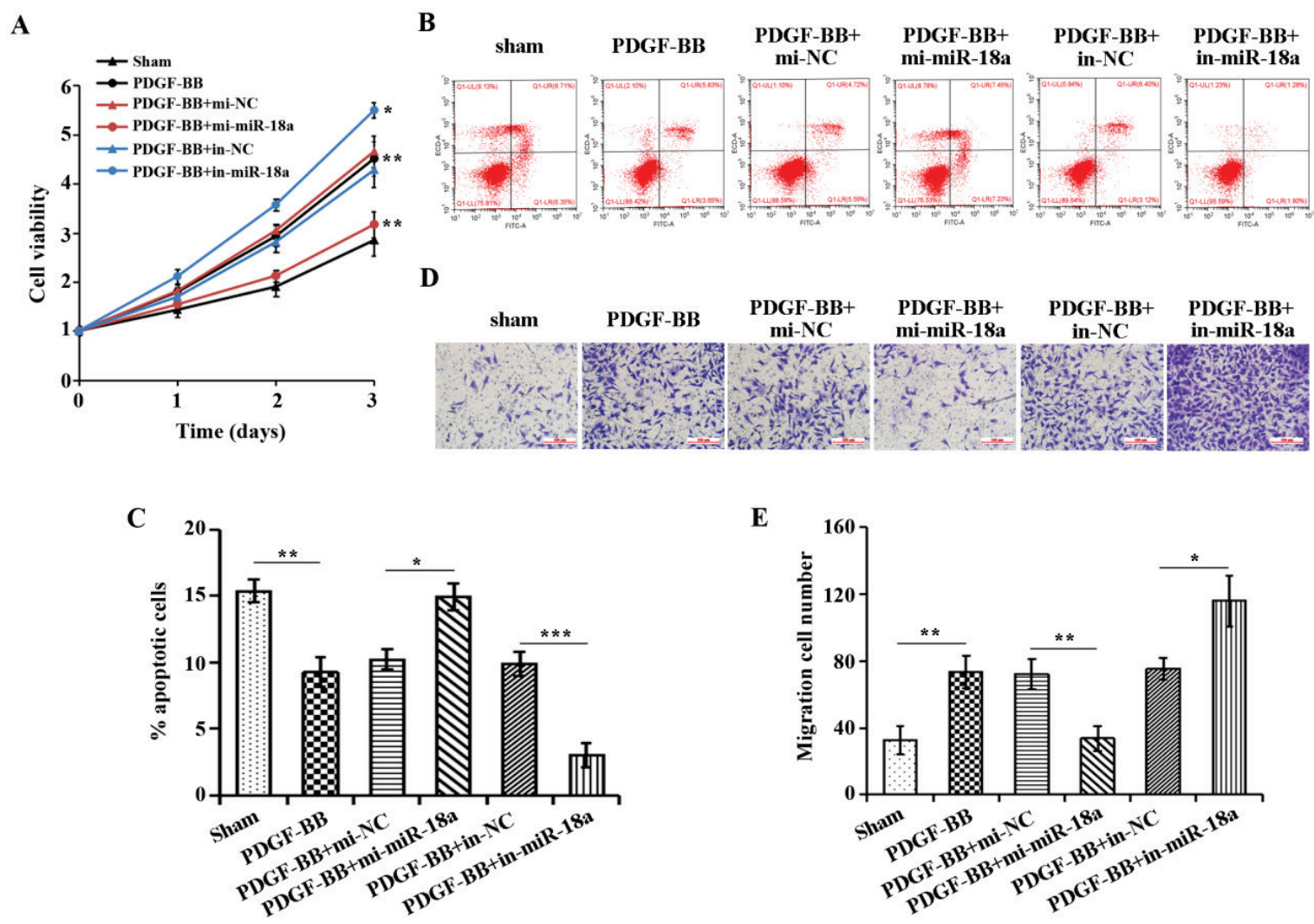

Fig. 2. Effect of over-expression or knockdown of miR-18a on proliferation, apoptosis and migration of ASMC intervened by PDGF-BB. A) MTT assay outcome on cell proliferation in each group, B) Flow cytometry detection of apoptosis in each treatment group, C) Quantitative analysis by flow cytometry. D) Transwell detection of cell migration in each treatment group. E) Quantitative analysis of transwell detection. $* \mathrm{P}<0.05, * * \mathrm{P}<0.01, * * * \mathrm{P}<0.001$.

PDGF-BB was used to treat ASMCs and intervene the expression of miR-18a. As presented by Fig. 1B, miR-18a expression in PDGF-BB group was drastically downregulated relative to the sham group $(\mathrm{P}<0.001)$, miR-18a expression in PDGF-BB + mi-miR-18a group was up-regulated comparing to $\mathrm{PDGF}-\mathrm{BB}+$ mi-NC $(\mathrm{P}<0.01)$, miR-18a expression in PDGF-BB + in-miR-18a group was down-regulated in comparison with PDGF-BB + in-NC $(\mathrm{P}<0.01)$. These results demonstrated that miR-18a expression was reduced in sputum from asthmatic patients and in ASMCs after PDGF-BB treatment.

MiR-18a inhibits PDGF-BB-induced proliferation and migration of ASMCs and promotes their apoptosis.

To investigate the role of miR-18a in ASMCs, we transfected miR-18a mimics and miR-18a inhibitor in ASMCs cells to over-express or disrupt miR-18a expression. MTT, flow cytometry and transwell were applied to examine the proliferation, apoptosis and migration ability of cells in each treatment group. The results showed (Fig. 2A-E) that PDGF-BB could promote the proliferation and migration of ASMCs and inhibit their apoptosis compared with the sham group. However, overexpression of miR-18a significantly alleviated the effects of PDGF-BB treatment on the proliferation, apoptosis and migration of ASMCs. Interference with miR-18a expression significantly indorsed the promoting effect of PDGF-BB treatment on the proliferation and migration of ASMCs and the inhibitory effect on apoptosis. These outcomes confirmed that PDGF-BB promoted PDGF-BB cell proliferation and migration as well as suppressed apoptosis, while miR-18a suppressed cell proliferation and migration as well as promoted apoptosis.

MiR-18a attenuates the inhibitory effect of PDGF-BBinduced vasoconstrictor phenotype proteins in ASMCs

We then assessed the effect of miR-18a on PDGF-BB-induced phenotypic changes in ASMCs by detecting the expression of vasoconstrictor phenotype marker proteins by Western blot. Figure 3 showed that the expressions of SM-22 $\alpha, \alpha$-SM-actin, and calponin were significantly up-regulated in the PDGF-BB group relative to the sham group, $\mathrm{SM}-22 \alpha, \alpha$-SM-actin, and 
calponin were significantly down-regulated in the PDGF$\mathrm{BB}+$ mi-miR-18a group in comparison to the PDGF-BB + mi-NC, and SM-22 $\alpha, \alpha-S M-a c t i n$, and calponin were up-regulated in the PDGF-BB + in-miR-18a group comparing to the PDGF-BB + in-NC.

MiR-18a inhibits PDGF-BB-induced activation of the PI3K/AKT signaling pathway in ASMCs

To investigate the mechanism of miR-18a in ASMCs, the protein expression of key molecules of the PI3K/AKT signaling pathway was detected by western blot. Figure 4 illustrated that compared with the sham group, the ratios of $\mathrm{p}-\mathrm{PI} 3 \mathrm{~K} / \mathrm{PI} 3 \mathrm{~K}$ and $\mathrm{p}-\mathrm{AKT} / \mathrm{AKT}$ were significantly up-regulated in the PDGF-BB group, in comparison to PDGF-BB + mi-NC, the ratios were significantly down-regulated in the PDGF-BB + mi-miR18a group, and comparing to PDGF-BB + in-NC, the ratios were up-regulated in the PDGF-BB + in-miR-18a group. These outcomes suggest that PDGF-BB can promote proliferation and migration, as well as inhibit apoptosis by activating the PI3K/AKT pathway, while miR-18a can block the process.
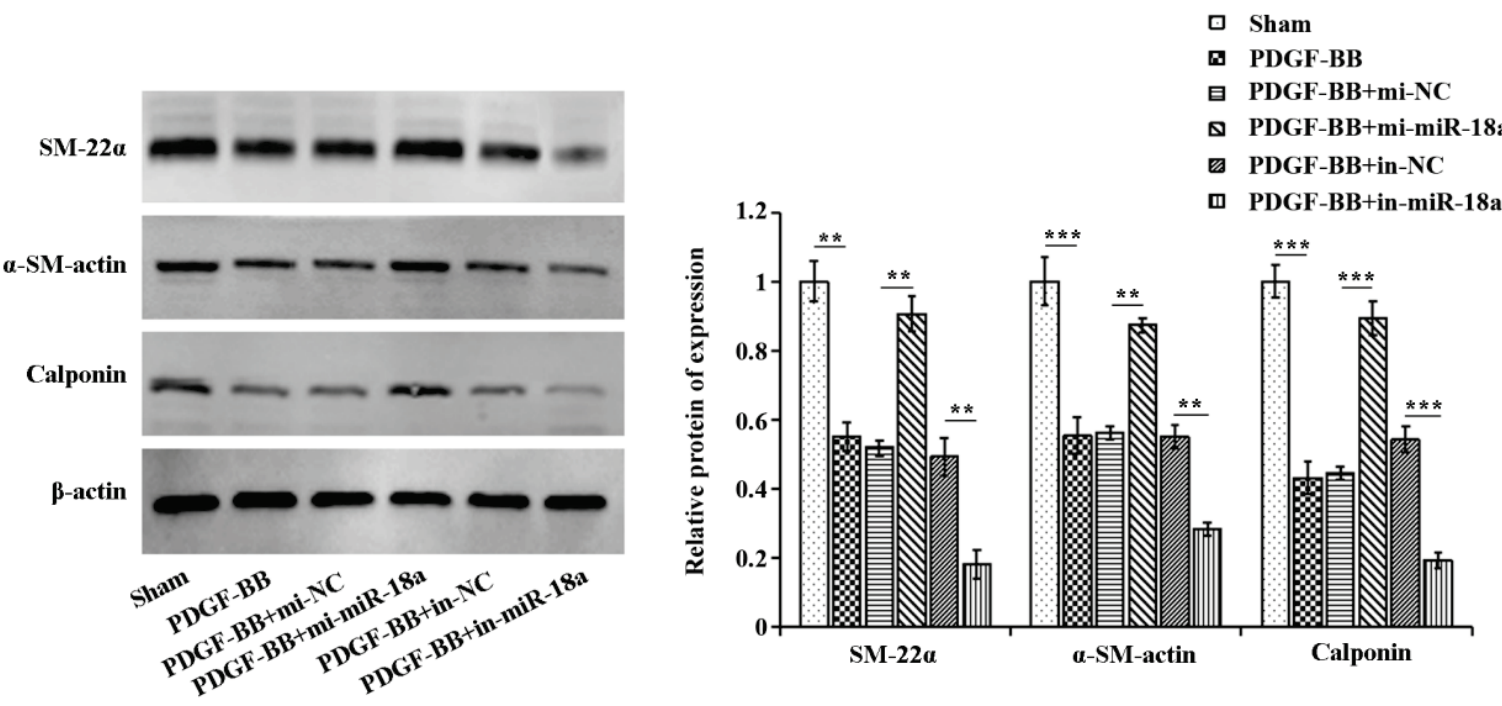

Fig. 3. MiR-18a elevates the expression of vasoconstrictor phenotypic markers in PDGF-BB-induced ASMCs. Western blot was used to examine the protein expression of SM-22a, a-SM-actin, and calponin in cells of each treatment group. $* * \mathrm{P}<0.01, * * * \mathrm{P}<0.001$.
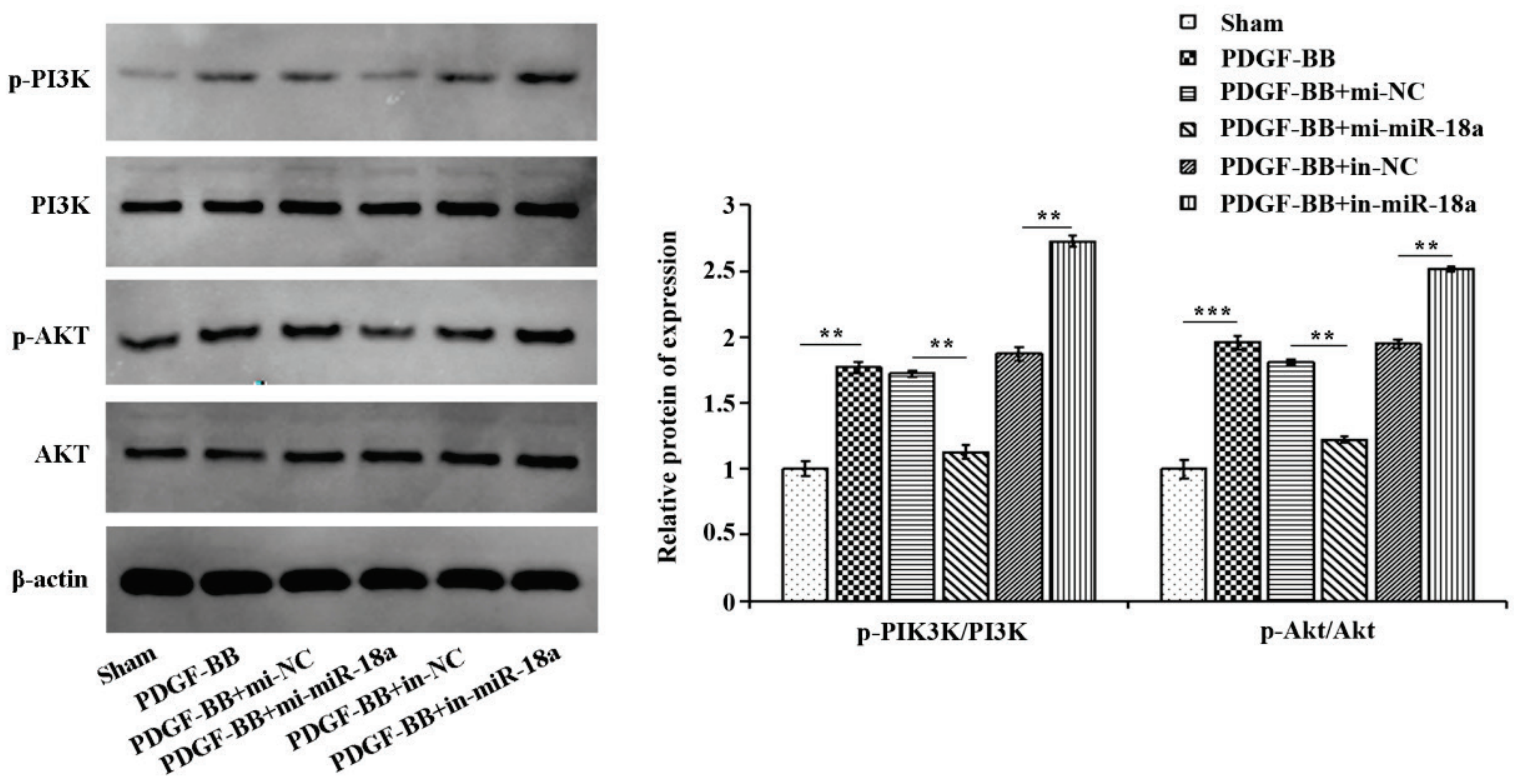

Fig. 4. MiR-18a inhibits PDGF-BB-induced activation of $P I 3 K / A K T$ signaling pathway in ASMCs. Western blot for investigating the expression of PI3K, p-PI3K, AKT and p-AKT in each treatment group. ${ }^{* * P}<0.01, * * * P<0.001$. 
A

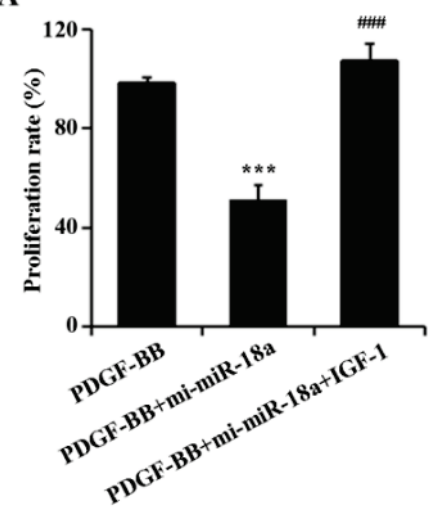

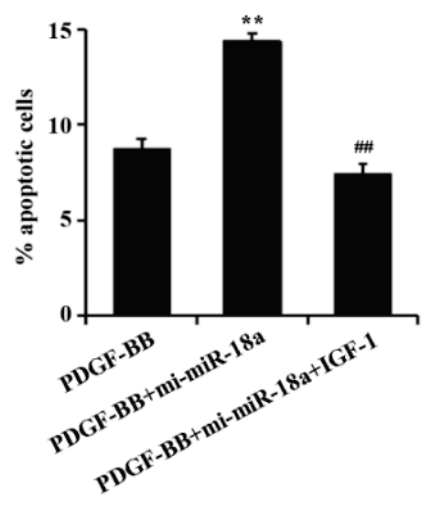

C

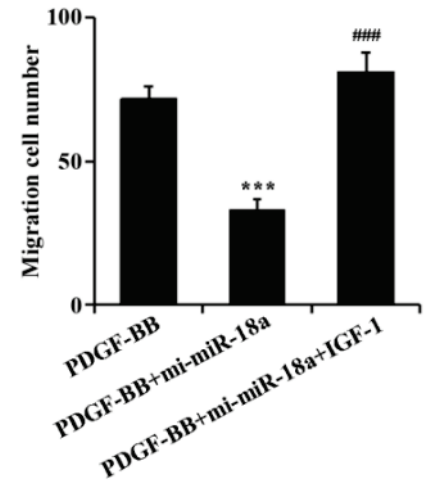

D
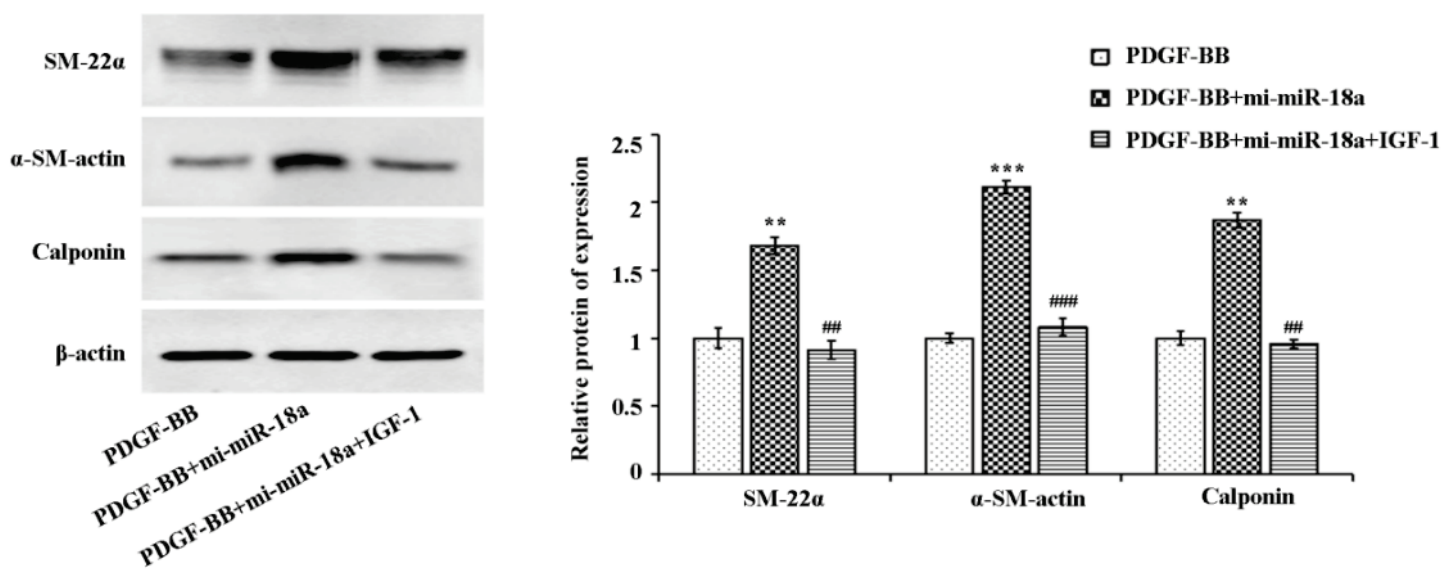

Fig. 5. PI3K/AKT signaling pathway agonist inhibited miR-18a-induced interference on PDGF-BB-related proliferation, apoptosis, migration and phenotypic transformation of ASMCs. A) MTT assay examination on the changes in cell proliferation ability of each treatment group, B) Flow cytometry outcome on the apoptosis of each treatment group, C) Transwell detection of the changes in cell migration ability of each treatment group, D: Western blot results on the expression levels of SM-22a, a-SM-actin, and calponin in cells of each treatment group, D) Quantitative analysis of gray values of SM-22a, a-SM-actin, and calponin bands, respectively. ${ }^{* * P}<0.01$, ${ }^{* * *} \mathrm{P}<0.001$ vs. PDGF-BB group. ${ }^{\# \# P}<0.01,{ }^{\# \#} \mathrm{P}<0.001$ vs. PDGF-BB+mi-miR-18a group.

To further validate that miR-18a is inhibiting airway remodeling by preventing PI3K/AKT signaling pathway activation, the PI3K/AKT signaling pathway agonist IGF-1 for reversion experiments was used. The experimental results showed (Fig. 5A-C) that IGF-1 reversibly over-expressed miR-18a on PDGF-BBinduced proliferation, apoptosis and migration of ASMCs. Figure 5D showed that miR-18a increased the expression of contractile phenotype marker proteins (SM-22 $\alpha, \alpha-S M-a c t i n$, calponin) in PDGF-BB-induced ASMCs, while IGF-1 reversed the expression of the above proteins. These outcomes together confirmed that miR-18a exerts an inhibitory effect on airway remodeling by restraining the PI3K/AKT signaling pathway.

\section{Discussion}

Airway remodeling in bronchial asthma is a series of chronic airway structural changes including airway wall thickening, mucosal epithelial injury, subepithelial fibrosis, increased extracellular matrix deposition, goblet cell metaplasia, smooth muscle cell proliferation, and revascularization (Fehrenbach et al. 2017, Hirota and Martin 2013, Kaczmarek et al. 2019). Studies have shown that airway remodeling occurs in the early stage of asthma, without effective clinical prevention and treatment in the irreversible late stage. Therefore, the early and effective intervention of irreversible airway lesions is an urgent challenge. Airway smooth muscle can proliferate under the activation of cytokines (such as PDGF, VEGF, EGF and TGF- $\beta$, etc.), accompanied by increased cell migration (Boulet 2018). It has been confirmed that the proliferation and migration ability of airway smooth muscle of asthmatic patients in vitro is comparable to or higher than that of smooth muscle in response to cytokines such as PDGF, and significantly higher than the level in normal airway smooth muscle (Munakata 2006). Therefore, in this study, 
PDGF-BB was used to stimulate ASMCs and to investigate their phenotypic changes upon cytokine stimulation and the regulation of cell proliferation and migration by miR-18a. It has been reported that miR-18a expression decreased in nasal biopsies of asthma patients (Zhao et al. 2018), and the results of this study are consistent with those reported in the articles. We have found that the miR-18a expression decreased in the sputum of asthma patients. The cellular miR-18a expression drastically decreased after PDGF-BB induction of ASMCs. The elevation in proliferation and migration of ASMCs is a key process in asthma-related airway remodeling (Ammit and Panettieri 2003). PDGF$\mathrm{BB}$ can induce proliferation and migration of ASMCs, and the apoptotic ability is thus attenuated. Further overexpression of miR-18a inhibited the effect of PDGF-BB on the proliferation and migration of ASMCs. This shows that miR-18a is involved in regulating the role of ASMCs in airway remodeling.

ASMCs phenotype modulation is mainly reversible conversion between contractile and proliferative phenotypes (Wright et al. 2013). ASMCs express a variety of proteins, such as $\alpha$-SM-actin, SM-22 $\alpha$, calponin, in the contractile phenotype. When ASMCs cells switch to a synthetic phenotype, contractile phenotype proteins $\alpha$-SM-actin, SM- $22 \alpha$, and calponin expression decrease, and cells begin to proliferate and migrate. This study showed that $\alpha$-SM-actin, SM-22 $\alpha$, and calponin protein expression was significantly lower in ASMCs under the induction of PDGF-BB. However, overexpression of miR-18a promoted the expression of contractile proteins.

In recent years, the role of the PI3K/AKT signaling pathway on airway remodeling has attracted much attention. Studies have shown that the activation of the PI3K/AKT signaling pathway is closely related to the proliferation of AMSCs (Liu et al. 2018, Shao et al. 2019, Zhao et al. 2018), that is, the activation of the PI3K signaling pathway can cause airway remodeling. It has been shown that targeting PI3K signaling significantly inhibits fibroblast expansion and fibrotic remodeling (Campa et al. 2018). In this study, PDGF-BB induced $\mathrm{PI} 3 \mathrm{~K} / \mathrm{AKT}$ pathway activation in ASMCs, while miR-18a inhibited the PI3K/AKT pathway. Recovery experiments confirmed that miR-18a inhibited the proliferation and migration of AMSCs and ECM protein production by inhibiting the PI3K/AKT pathway through IGF-1, an activator of the PI3K/AKT pathway. These results were consistent with those reported in the literature.

In summary, miR-18a expression was significantly down-regulated in proliferating ASMCs as proven by this study. Its high expression was able to suppress the activation of PI3K/AKT pathway, thereby alleviating PDGF-BB stimulation-induced proliferation and migration of ASMCs. Our study provides important new insights into the study of the molecular mechanism of miR-18a in asthma. The pathogenesis of asthma is closely related to airway remodeling. The targeted overexpression of miR-18a in ASMCs may provide a potential novel therapeutic approach for inhibiting airway remodeling associated with asthma pathogenesis.

\section{Conflict of Interest}

There is no conflict of interest.

\section{References}

AMMIT AJ, PANETTIERI RJ: Airway smooth muscle cell hyperplasia: a therapeutic target in airway remodeling in asthma? Prog Cell Cycle Res 5: 49-57, 2003.

BOULET LP: Airway remodeling in asthma: update on mechanisms and therapeutic approaches. Curr Opin Pulm Med 24: 56-62, 2018. https://doi.org/10.1097/MCP.0000000000000441

BRETHERTON R, BUGG D, OLSZEWSKI E, DAVIS J: Regulators of cardiac fibroblast cell state. Matrix Biol 91-92: 117-135, 2020. https://doi.org/10.1016/j.matbio.2020.04.002

CAMPA CC, SILVA RL, MARGARIA JP, PIRALI T, MATTOS MS, KRAEMER LR, REIS DC, GROSA G, COPPERI F, DALMARCO EM, LIMA-JUNIOR R, APRILE S, SALA V, DAL BELLO F, PRADO DS, ALVES-FILHO JC, MEDANA C, CASSALI GD, TRON GC, TEIXEIRA MM, CIRAOLO E, RUSSO RC, HIRSCH E: Inhalation of the prodrug PI3K inhibitor CL27c improves lung function in asthma and fibrosis. Nat Commun 9: 5232, 2018. https://doi.org/10.1038/s41467-018-07698-6

DAI Y, LI Y, CHENG R, GAO J, LI Y, LOU C: TRIM37 inhibits PDGF-BB-induced proliferation and migration of airway smooth muscle cells. Biomed Pharmacother 101: 24-29, 2018. https://doi.org/10.1016/j.biopha.2018.02.057 
FEHRENBACH H, WAGNER C, WEGMANN M: Airway remodeling in asthma: what really matters. Cell Tissue Res 367: 551-569, 2017a. https://doi.org/10.1007/s00441-016-2566-8

GUIDA G, RICCIO AM: Immune induction of airway remodeling. Semin Immunol 46: 101346, 2019. https://doi.org/10.1016/j.smim.2019.101346

HIROTA N, MARTIN JG: Mechanisms of airway remodeling. Chest 144: 1026-1032, 2013. https://doi.org/10.1378/chest.12-3073

INUI M, MARTELLO G, PICCOLO S: MicroRNA control of signal transduction. Nat Rev Mol Cell Biol 11: 252-263, 2010. https://doi.org/10.1038/nrm2868

KACZMAREK KA, CLIFFORD RL, KNOX AJ: Epigenetic Changes in Airway Smooth Muscle as a Driver of Airway Inflammation and Remodeling in Asthma. Chest 155: 816-824, 2019. https://doi.org/10.1016/j.chest.2018.10.038

KING-BIGGS MB: Asthma. Ann Intern Med 171: C49-C64, 2019. https://doi.org/10.7326/AITC201910010

LIU Y, MIAO Y, GAO X, WANG YY, WANG H, ZHENG YW, ZHAO ZY: MicroRNA-200a Affects the Proliferation of Airway Smooth Muscle Cells and Airway Remodeling by Targeting FOXC1 via the PI3K/AKT Signaling Pathway in Ovalbumin-Induced Asthmatic Mice. Cell Physiol Biochem 50: 2365-2389, 2018. https://doi.org/10.1159/000495097

LOU L, TIAN M, CHANG J, LI F, ZHANG G: MiRNA-192-5p attenuates airway remodeling and autophagy in asthma by targeting MMP-16 and ATG7. Biomed Pharmacother 122: 109692, 2020. https://doi.org/10.1016/j.biopha.2019.109692

MICHALIK M, WOJCIK-PSZCZOLA K, PAW M, WNUK D, KOCZURKIEWICZ P, SANAK M, PEKALA E, MADEJA Z: Fibroblast-to-myofibroblast transition in bronchial asthma. Cell Mol Life Sci 75: 3943-3961, 2018. https://doi.org/10.1007/s00018-018-2899-4

MUNAKATA M: Airway remodeling and airway smooth muscle in asthma. Allergol Int 55: 235-243, 2006. https://doi.org/10.2332/allergolint.55.235

PUA HH, ANSEL KM: MicroRNA regulation of allergic inflammation and asthma. Curr Opin Immunol 36: 101-108, 2015. https://doi.org/10.1016/j.coi.2015.07.006

SAMITAS K, CARTER A, KARIYAWASAM HH, XANTHOU G: Upper and lower airway remodelling mechanisms in asthma, allergic rhinitis and chronic rhinosinusitis: The one airway concept revisited. Allergy 73: 993-1002, 2018. https://doi.org/10.1111/all.13373

SHAO Y, CHONG L, LIN P, LI H, ZHU L, WU Q, LI C: MicroRNA-133a alleviates airway remodeling in asthtama through PI3K/AKT/mTOR signaling pathway by targeting IGF1R. J Cell Physiol 234: 4068-4080, 2019. https://doi.org/10.1002/jep. 27201

SUOJALEHTO H, LINDSTROM I, MAJURI ML, MITTS C, KARJALAINEN J, WOLFF H, ALENIUS H: Altered microRNA expression of nasal mucosa in long-term asthma and allergic rhinitis. Int Arch Allergy Immunol 163: 168-178, 2014. https://doi.org/10.1159/000358486

VISHNOI A, RANI S: MiRNA Biogenesis and Regulation of Diseases: An Overview. Methods Mol Biol 1509: 1-10, 2017. https://doi.org/10.1007/978-1-4939-6524-3_1

WRIGHT DB, TRIAN T, SIDDIQUI S, PASCOE CD, JOHNSON JR, DEKKERS BG, DAKSHINAMURTI S, BAGCHI R, BURGESS JK, KANABAR V, OJO OO: Phenotype modulation of airway smooth muscle in asthma. Pulm Pharmacol Ther 26:42-49, 2013. https://doi.org/10.1016/j.pupt.2012.08.005

YANG H, LI W, ZHANG Y, LI M, GAO Y, LAO C, SHI B: Regulatory role of miR-18a to CCN2 by TGF-beta1 signaling pathway in pulmonary injury induced by nano- $\mathrm{SiO}_{2}$. Environ Sci Pollut Res Int 25: 867-876, 2018. https://doi.org/10.1007/s11356-017-0344-0

YE F, TIAN L, ZHOU Q, FENG D: LncRNA FER1L4 induces apoptosis and suppresses EMT and the activation of PI3K/AKT pathway in osteosarcoma cells via inhibiting miR-18a-5p to promote SOCS5. Gene 721: 144093, 2019. https://doi.org/10.1016/j.gene.2019.144093

YUAN J, TAN L, YIN Z, ZHU W, TAO K, WANG G, SHI W, GAO J: MIR17HG-miR-18a/19a axis, regulated by interferon regulatory factor-1, promotes gastric cancer metastasis via Wnt/beta-catenin signalling. Cell Death Dis 10: 454, 2019. https://doi.org/10.1038/s41419-019-1685-Z 
ZHANG C, WANG J, MA X, WANG W, ZHAO B, CHEN Y, CHEN C, BIHL JC: ACE2-EPC-EXs protect ageing ECs against hypoxia/reoxygenation-induced injury through the miR-18a/Nox2/ROS pathway. J Cell Mol Med 22: 1873-1882, 2018. https://doi.org/10.1111/jcmm.13471

ZHAO X, YU FQ, HUANG XJ, XU BY, LI YL, ZHAO XY, GUO HF, LUAN B: Azithromycin influences airway remodeling in asthma via the PI3K/Akt/MTOR/HIF-1alpha/VEGF pathway. J Biol Regul Homeost Agents 32: 1079-1088, 2018. 